\title{
Prospective Assessment of Direction of Cervical Canal in Routine Hysteroscopy
}

\author{
Divyesh V. Shukla1, Shilpi D. Shukla' ${ }^{1}$, Sangita Patel2 ${ }^{2}$, Amit Shah ${ }^{3}$ \\ ${ }^{1}$ Department of Obg/Gyn and Minimal Access Surgery, Isha Hospital, Sarabhai Campus, Opposite Atlantis Mall, Vadodara, India \\ ${ }^{2}$ Department of PSM, Govt Medical College Baroda, Vadodara, India \\ ${ }^{3}$ Isha Hospital, Vadodara, India \\ Email: divyeshshukla@yahoo.com, shuklshilpi363@yahoo.in, sangita_psm@yahoo.co.in, amit20668@gmail.com
}

How to cite this paper: Shukla, D.V., Shukla, S.D., Patel, S. and Shah, A. (2021) Prospective Assessment of Direction of Cervical Canal in Routine Hysteroscopy. Open Journal of Obstetrics and Gynecology, 11, 660-673.

https://doi.org/10.4236/ojog.2021.116062

Received: April 26, 2021

Accepted: June 7, 2021

Published: June 10, 2021

Copyright $\odot 2021$ by author(s) and Scientific Research Publishing Inc. This work is licensed under the Creative Commons Attribution International License (CC BY 4.0).

http://creativecommons.org/licenses/by/4.0/

\begin{abstract}
Introduction: If the direction of the cervical canal is known, the commonly performed procedures as sono salpingography, embryo transfer, IUD insertion, cervical dilation etc. will be easier. The reported resultant trauma to the cervical canal and uterus during these procedures also can be avoided. As we know the cervical canal is tortuous in majority of cases, but the exact course is not yet reported or known. Objective: The objective of the present study was to try to identify the various directions of cervical canal while performing routine hysteroscopic surgeries. Methods: Four-point cervical canal direction was assessed while performing routine hysteroscopic procedures using $5 \mathrm{fr}$ Bettocchi operative assembly with $2.9 \mathrm{~mm} \mathrm{12-degree} \mathrm{telescope} \mathrm{(Karl-Storz).}$ The study group was patients with infertility who required hysteroscopy and laparoscopic evaluation as per infertility treatment protocol or else required hysteroscopy for AUB. The study was carried out at tertiary care referral hospital for minimal access surgeries for a period was of $2 \mathrm{yr} .9$ months year from May 2017 to Feb 2020. Results: Down-right or left-up-straight combination of movement (DRUS, DLUS) was the most common cervical direction found in $72 \%$ patients. If DURS (down-up-right-straight) movement is added these 3 movements together are seen in about $82 \%$ of patients. No cervical dilation is required when $5 \mathrm{fr}$ hysteroscopic assembly was used in study group. No operative complications were found. Conclusion: DRUS and DLUS (down-right or left-up and straight) combination of movements are most frequent direction of cervical canal observed in $72 \%$ of patients.
\end{abstract}

\section{Keywords}

Cervical Canal Direction, 5 fr Bettocchi Hysteroscope, Down, Right, Up, Straight 


\section{Introduction}

The direction of the cervix is variable. Procedures involving the need for cervical dilation as dilation and curettage procedure or hysteroscopy or other procedure involving the use of blind insertion of the cannula as in sono salpingography, embryo transfer, IUD insertion can result in cervical or uterine injury. Many authors have suggested various methods to overcome the difficult cervical entry. [1] This published study mentioned only one point, while visually entering cervix, position hysteroscope in cervical canal so as to maintain canal opening in the center of picture. No mention regarding direction of the cervical canal was made. There is no publication describing the cervical canal direction in the literature even when the publications are for difficult cervical entry. Therefore, it is very much necessary to know the usual cervical direction by various methods in women. The literature has limited information, or no article published in this matter.

So, we decided to assess the direction of the cervical canal by 4 point direction assessment while performing routine hysteroscopic procedures without carrying out cervical dilatation.

\section{Aims and Objectives}

1) To note the 4-point direction of the cervical canal while performing diagnostic hysteroscopies and hysteroscopic surgeries.

2) To note the requirement of dilation of the cervix when $5 \mathrm{~mm}$ diameter of Bettocchi operating channel (15 fr) was used for diagnostic or operative procedures.

3) To find out how many patients required gentle push forward at internal OS before hysteroscope finally entered uterine cavity or scissors were used to cut adhesions at internal OS.

\section{Material and Methods}

The study was approved by an institutional ethical committee. (Isha Hospital Ethical committee)

This prospective study was carried out for a period of 2 yrs. 9 months from May 2017 to Feb 2020. All patients who underwent diagnostic and operative hysteroscopic surgeries were included in the study. The patients who required operative hysteroscopy using 22 fr resectoscope, patients having congenital malformations other than septate uterus, and all patients of asherman's syndrome except type 3 were excluded from the study.

During the study period there were total 392 patients in whom hysteroscopy was performed. 142 patients were excluded from the study in whom $22 \mathrm{fr}$ resectoscope was used to treat pathology or patients who had congenital malformations other than septate uterus and asherman's syndrome except type 3 (cervical stenosis at internal OS). [2]

The remaining 250 patients were included in the study after taking informed 
consent. Out of these $86 \%$ of patients had infertility in whom hysteroscopy and laparoscopy was performed, in remaining patients hysteroscopy were performed for AUB (abnormal uterine bleeding). Patients with all genital tract malformations other than septate uterus and all asherman's syndrome except type 3 (cervical stenosis at internal OS) were excluded. [2]

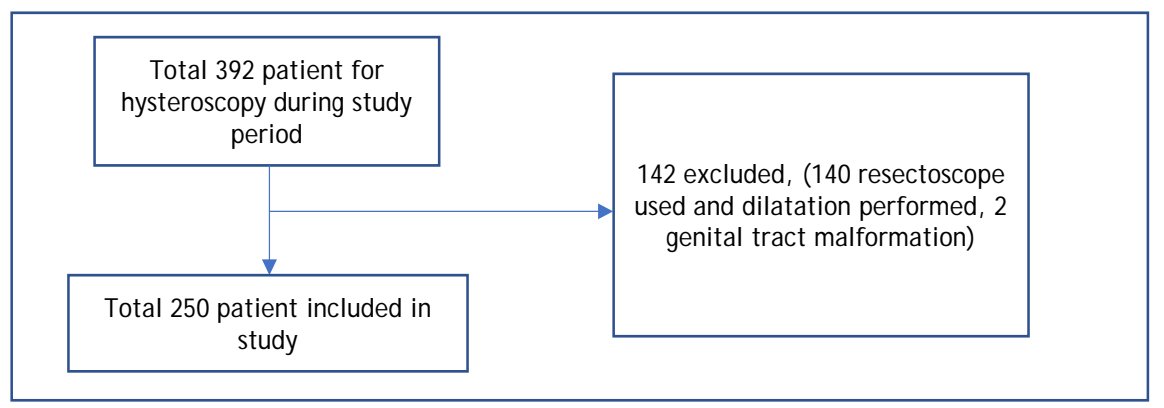

The study was carried out in the tertiary care referral department of minimal access surgery, Isha Hospital, Vadodara. Detail history was taken which included the history of past surgeries performed. Pre-operative general examination and PS/PV examination was done. TVS was performed for pelvic pathologies. All patients underwent standard preoperative investigations and anaesthesia risk assessment before the surgery.

\section{Method}

After giving general anaesthesia first procedure performed was hysteroscopy.

\section{Method to perform hysteroscopy:}

Operation theatre table was kept horizontal and hysteroscopy/Laparoscopy was performed in dorsal lithotomy position with thighs flexed about 15 degree and popliteal fossa well supported in lithotomy stirrups. Surgeries were performed by a single surgeon having experience of performing large number of hysteroscopic surgeries and has experience in training obstetricians and gynaecologists in these procedures for the last 15 years.

For performing diagnostic or operative hysteroscopies Karl storz Bettocchi made operative hysteroscopy assembly with oval sheath was used with $2.9 \mathrm{~mm}$ 12 -degree telescope. It is a $5 \mathrm{~mm}$ diameter (15 fr) assembly with inner sheath of $4.3 \mathrm{~mm}$ with a semirigid working channel of $5 \mathrm{fr}$ and an outer sheath of 5 $\mathrm{mm}$ to be used with an inner sheath, Close system was used for hysteroscopy using normal saline and Endomat suction irrigation system made by Karl storz.

Visual entry was practiced while using operative hysteroscopic sheath and no cervical dilation was performed at the beginning of the procedure. The requirement of cervical dilation was noted before hysteroscopy.

The anterior lip of the cervix was held with allis tissue holding forceps or vulsellum forceps to stabilize. No traction was applied on the cervix in any direction. Dynamic change of intrauterine pressures was made during the procedure 
depending upon the requirement. In some patients high pressures (up to 150 mmhg) were used to maintain vision while in the cervical canal, which was decreased as soon as the uterine cavity was entered. External cervical OS of the cervix was occluded with allis forceps to decrease trans cervical leak in very few patients. (after entering uterine cavity).

To begin the tip of hysteroscopic assembly was positioned at external OS. Light cord was at kept at 12 o'clock position. Hysteroscope is now inserted through an external OS. The direction of the cervical canal noted was as per the movements of the tip of hysteroscopic assembly. As a part of standard method posterior wall of the cervical canal was followed while advancing the hysteroscope through the cervical canal in a way to have the cervical canal opening (relative dark area ) visualized more anterior or visible in the center of video streaming on screen. The light cord was rotated on the left when the hysteroscope is moved to in the direction to the right. The cord too is rotated to accommodate the cervical canal according to the available width of the cervical canal. The hysteroscope is generally advanced by changing the angle and rotating assembly. Mild resistance was overcome by a gentle push. The hysteroscopy channel is kept transverse in all while negotiating the internal OS and moving ahead of internal OS straight to finally enter the uterine cavity.

It was noted whether some force was required to finally enter the internal OS or scissors were used to cut adhesions at the internal OS (internal OS not seen).

The hysteroscopic sheath was angled up, down or rotated depending upon the uterine axis and treatment of pathology.

After hysteroscopy necessary laparoscopic surgery was performed as required.

The 4-point direction through which the hysteroscope was guided through the cervix to enter the uterine cavity was intimated to the assistant immediately. This direction of record was further verified by the same assistant from the video recording. This was immediately recorded as XXXX.

Common movements of the tip of hysteroscopic sheath performed were down, right, up, left, and straight. Various combinations of these movements to assess cervical direction numbered were of 21 types. False passages, uterine perforations, failure of a procedure or uterine inner wall injury resulting because of the procedure was noted. Complete records of the patient including patient profile, diagnosis, associated pathology, history of previous surgeries, associated medical condition, details of present surgery, and finally the intraoperative cervical direction records were entered in excel format and the data was further analyzed.

Descriptive statistics were used to describe various movement during hysteroscopy. Categorical variables were expressed as number (\%). Chi-square test was used to see an association between various combined directions and parity, age of the patient, previous surgery, and existing pathology.

Informed consent of the patients was taken. Institutional ethical committee approval was also taken. 


\section{Results}

Most common $1^{\text {st }}, 2^{\text {nd }}, 3^{\text {rd }}$, and $4^{\text {th }}$ individual 4 -point movement performed while performing hysteroscopy was, down 230 (92\%) in the first direction, right 199 (79.6\%) in the second direction, up 198 (79.2) in $3^{\text {rd }}$ direction, and straight 219 $(87.6 \%)$ in $4^{\text {th }}$ direction in the patients. The 5 th direction was on the left after the 1 st down movement, this was observed in 29 (11.6\%) of the patients (Figure 1-5, Table 1). These were individual movements performed while negotiating the cervical canal at different points.

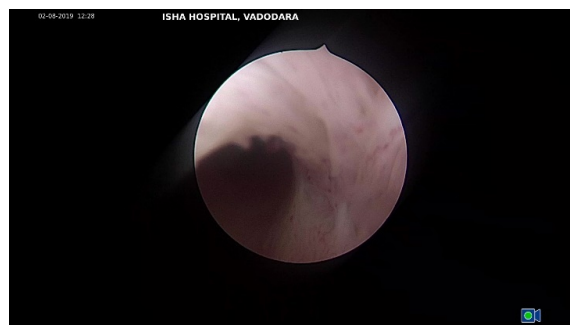

(a)

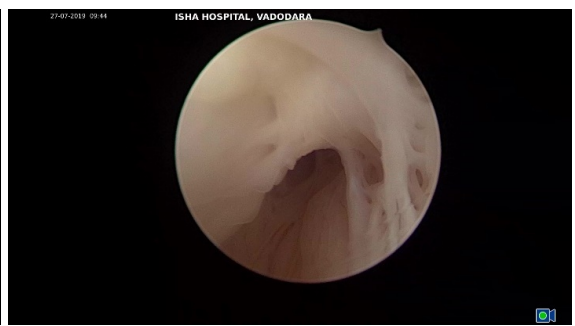

(b)

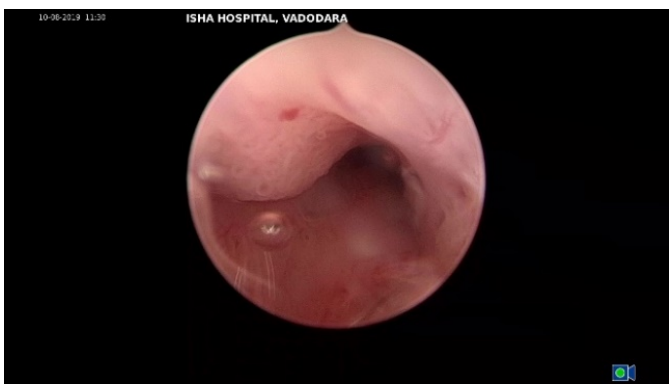

(c)

Figure 1. (a-c) Down (direction 1).

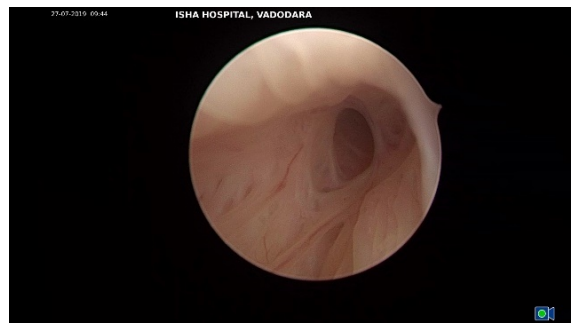

(a)

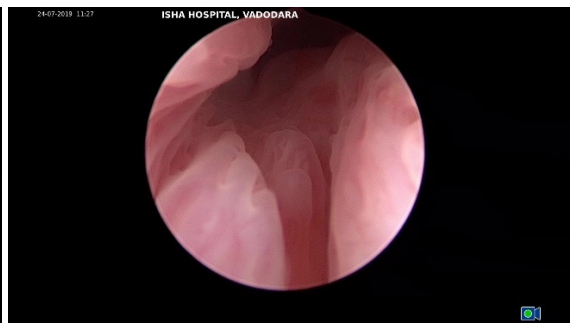

(b)

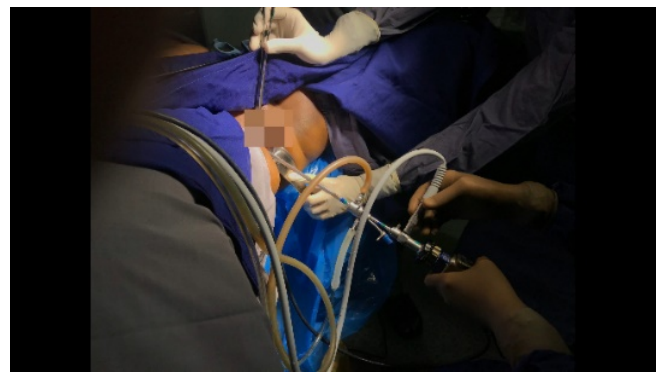

(c)

Figure 2. (a, b) Right (direction 2); (c) External picture of inner right up direction. 


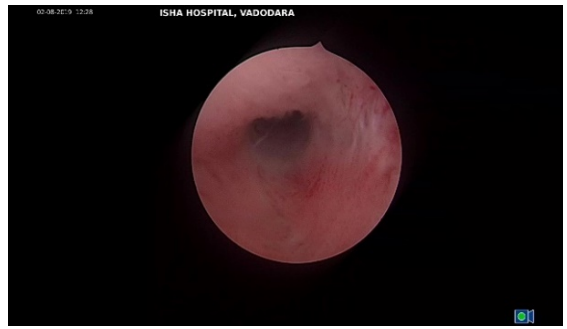

(a)

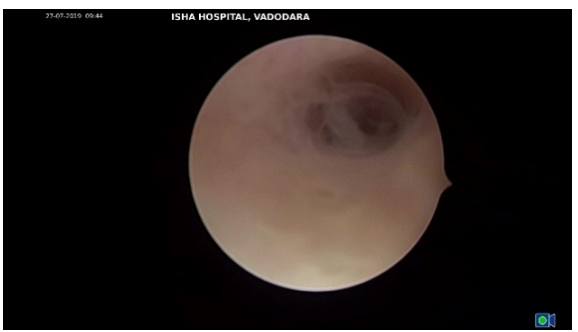

(b)

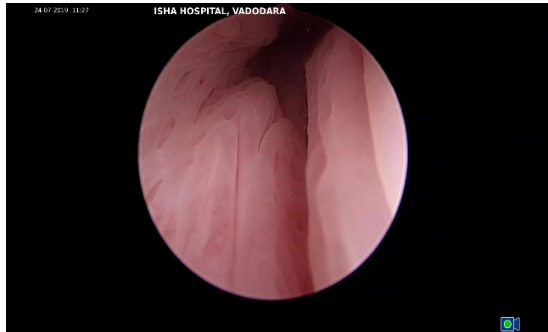

(c)

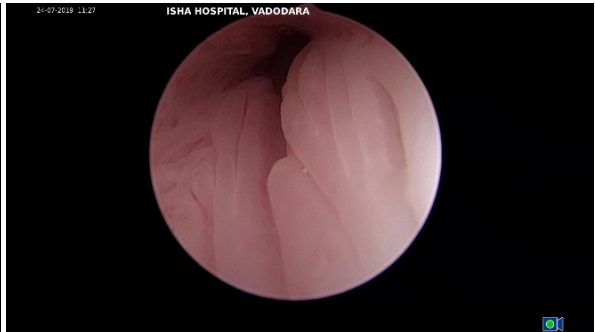

(d)

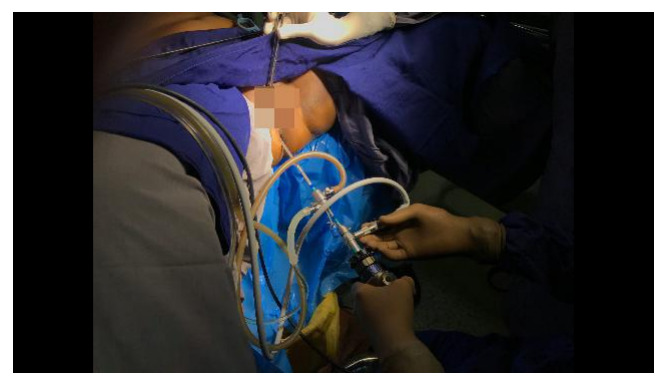

(e)

Figure 3. (a-d) Up (direction 3); (e) External picture of inner up direction.

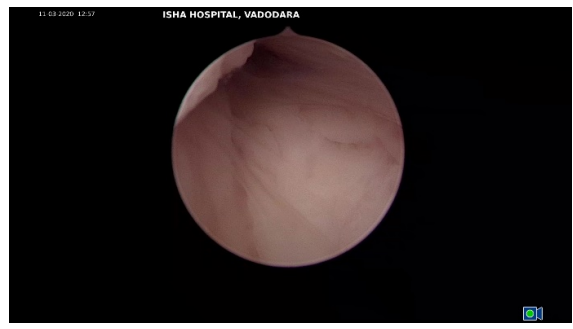

(a)

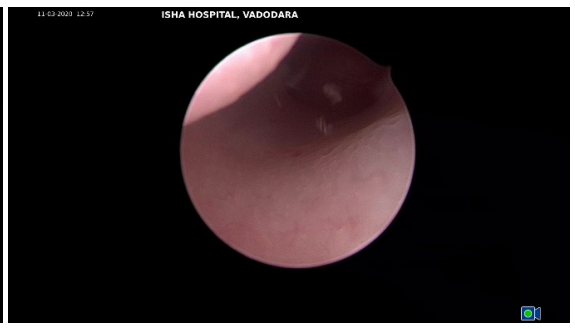

(b)

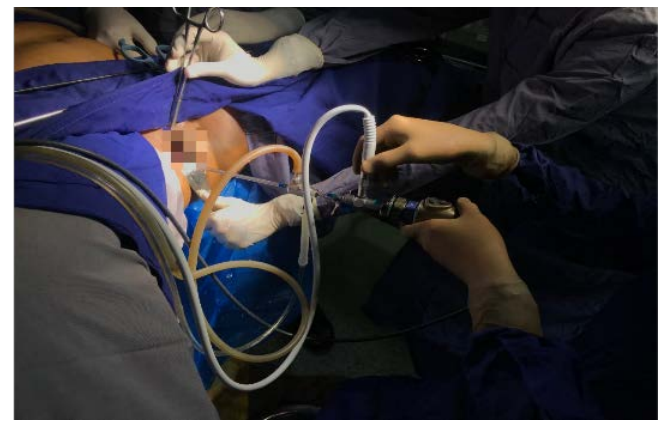

(c)

Figure 4. (a, b) Straight (direction 4); (c) External picture of inner final straight direction to enter internal OS of cervix. 


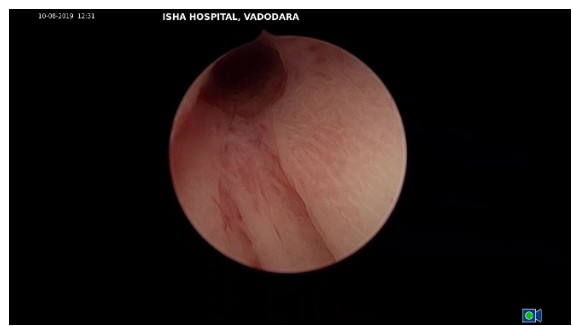

(a)

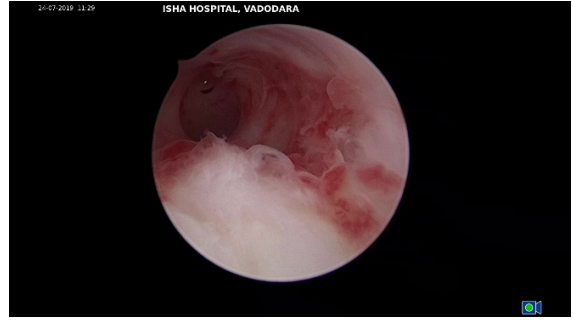

(c)

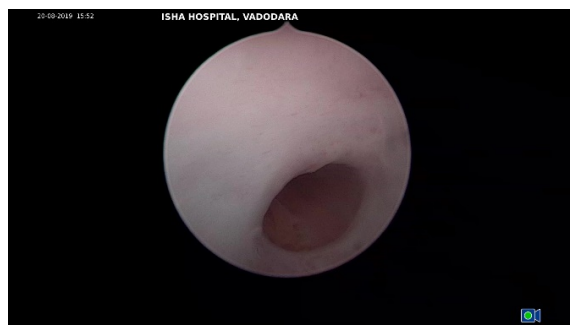

(e)

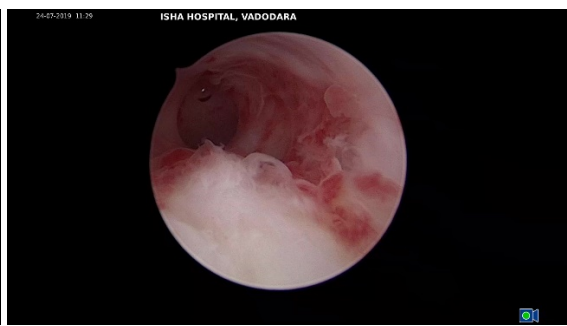

(b)

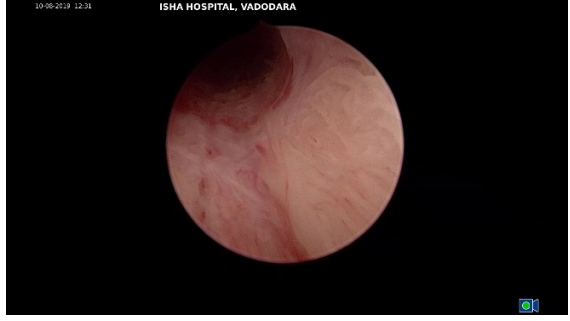

(d)

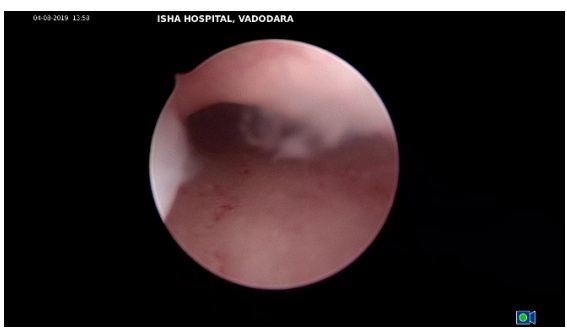

(f)

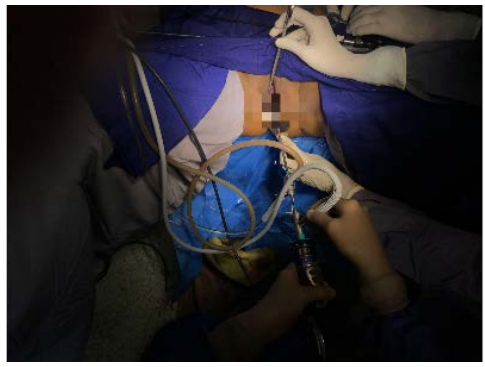

(g)

Figure 5. (a, b) After down to left (direction 5); (c-f) After down to left and up; (g) External picture UP.

Table 1. Individual directions of movement of the hysteroscopic sheath while in the cervical canal.

4-point Sequence of

direction of hysteroscopy First direction Second direction Third direction Fourth direction sheath while entering cervix

\begin{tabular}{lcccc}
\hline & No $(\%)$ & No $(\%)$ & No (\%) & No (\%) \\
\hline 1. Down & $230(92 \%)$ & $5(2 \%)$ & $0(0 \%)$ & $6(2.4 \%)$ \\
2. Right & $5(2 \%)$ & $199(79.6 \%)$ & $7(2.8 \%)$ & $0(0 \%)$ \\
3. UP & $0(0 \%)$ & $8(3.2 \%)$ & $198(79.2 \%)$ & $24(9.6 \%)$ \\
4. Straight & $7(2.8 \%)$ & $9(3.6 \%)$ & $36(14.4 \%)$ & $219(87.6 \%)$ \\
5. Left & $8(3.2 \%)$ & $29(11.6 \%)$ & $9(3.6 \%)$ & $1(0.4 \%)$ \\
\hline
\end{tabular}




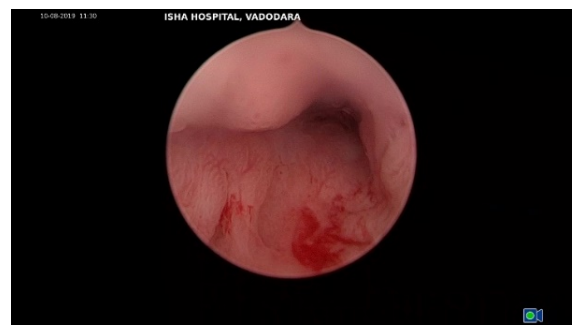

(a)

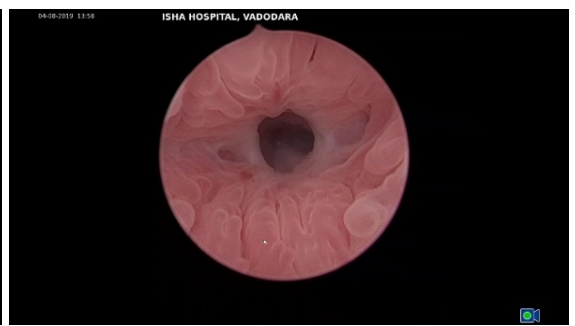

(b)

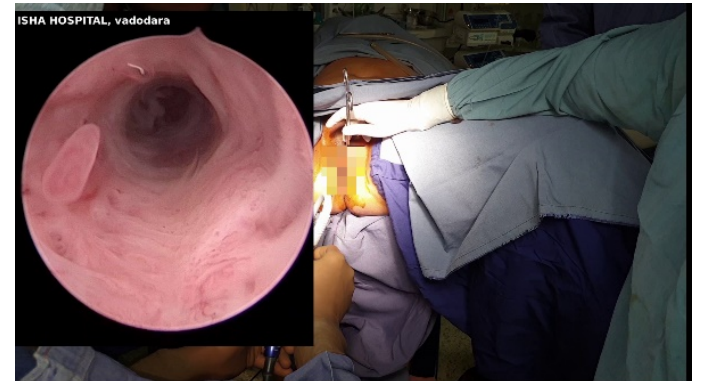

(c)

Figure 6. (a, b) Straight look of cervical canal from external OS; (c) Straight look of cervical canal from external OS with external view.

The most common movements performed were found to be significantly associated with the age of the patient. But they were not associated with parity, previous surgery, and associated pathology.

From the external OS to the internal OS straight cervical canal was observed only in $6(2.4 \%)$ patients (Table 2, Figure 6 ). The two most common combination of movements performed to enter uterine cavity was DRUS in 155 (62\%) (Figures 7(a)-(c)) and DLUS in 24 (9.6\%). (Table 2). DRUS, DLUS, and DURS combinations of movements were observed in $81.6 \%$ the patients. These combinations of movements indicated the direction of the cervical canal.

When the data of patient's age, diagnosis, history of previous surgery was analyzed, the same two most frequent direction of combinations of movements were noticed. This may prove that these variables do not affect the direction of cervical canal in general.

We have observed the cervix remains in its position even when there is a history of previous surgeries on the uterus or there is an associated pathology, the uterine axis changes beyond the internal OS.

The pathologies for which surgery was scheduled the study group of $250 \mathrm{pa}-$ tients were fibroid, cervical fibroid, adenomyoma endometriosis, and pelvic adhesions including tuberculosis. (215 (85.6\%)) In our study group, no patient had apical prolapse of the uterus.

Patients had the history of LSCS, myomectomy, Laparoscopy, and hysteroscopy performed in past and $\mathrm{D} \& \mathrm{C}$ as prior surgeries performed on the uterus. 76 (30.8\%) of patients had such history. Some of them had an associated history of appendectomy, laparotomy, Cholecystectomy, and splenectomy performed.

From the results, it is evident as the commonest observed combinations of 


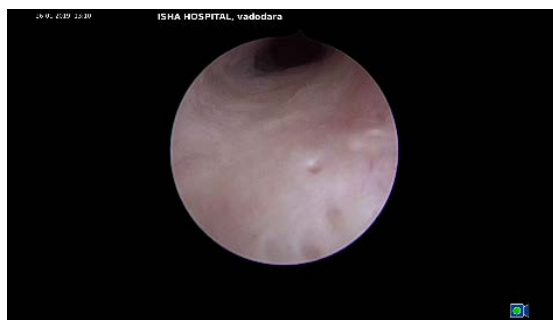

(a)

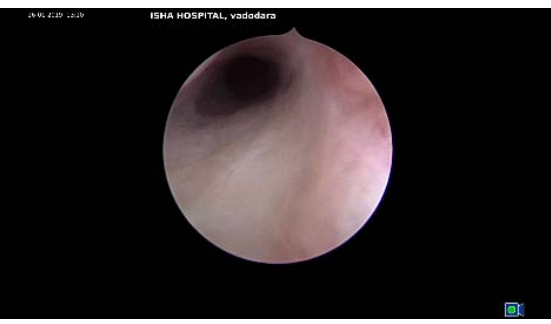

(b)

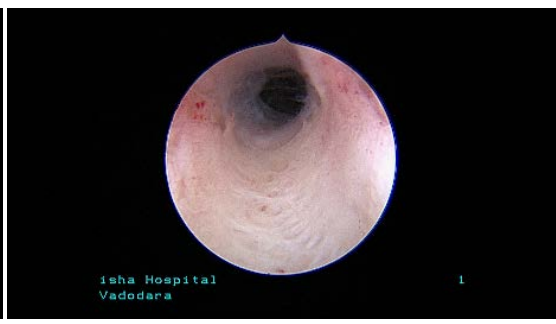

(c)

Figure 7. (a-c) DRUS panoramic view.

Table 2. Different combination of movements found to indicate a direction.

\begin{tabular}{|c|c|c|}
\hline No & Directions & No (\%) \\
\hline 1 & DRUS & $155(62 \%)$ \\
\hline 2 & DLUS & $24(9.6 \%)$ \\
\hline 3 & DRSU & $10(4 \%)$ \\
\hline 4 & DRSS & $8(3.2 \%)$ \\
\hline 5 & LRUS & $8(3.2 \%)$ \\
\hline 6 & SSSS & $6(2.4 \%)$ \\
\hline 7 & DRLS & $5(2 \%)$ \\
\hline 8 & DRUU & $5(2 \%)$ \\
\hline 9 & DUSS & $4(1.6 \%)$ \\
\hline 10 & DRLU & $4(1.6 \%)$ \\
\hline 11 & DRSD & $3(1.2 \%)$ \\
\hline 12 & RDUS & $3(1.2 \%)$ \\
\hline 13 & DURS & $2(0.8 \%)$ \\
\hline 14 & DSRU & $2(0.8 \%)$ \\
\hline 15 & DLUU & $2(0.8 \%)$ \\
\hline 16 & RUSD & $2(0.8 \%)$ \\
\hline 17 & DDSS & $2(0.8 \%)$ \\
\hline 18 & DLRS & $2(0.8 \%)$ \\
\hline 19 & DLRU & $1(0.4 \%)$ \\
\hline 20 & SSSL & $1(0.4 \%)$ \\
\hline 21 & DRUD & $1(0.4 \%)$ \\
\hline
\end{tabular}

D-Down, R-Right, U-UP, L-Left, S-Straight.

movements to indicate the cervical canal direction does not change with history of previous surgery performed or in presence of current pathology diagnosed for which the surgery was scheduled.*

No cervical dilation was required in these hysteroscopic procedures. Patients who had some resistance met at internal OS, a gentle straight push is made along the direction of the sheath, if the dark internal OS portion is visible. 
An interesting result was noted as in all patients who required gentle forward push to enter the uterine cavity, subsequent insertion was very easy without resistance at the internal OS. (Table 3)

In case of fibrosis visible at the internal OS sharp scissors is used to cut adhesions in appropriate directions to open the internal OS before the hysteroscopic sheath is made to enter the uterine cavity. (Figures 8(a)-(c)) In this study about $44(17.6 \%)$ patients had resistance met at internal OS.

Gentle push at the internal OS resulted in endometrial disruption in 5 cases, 2 in the anterior wall, 2 in the posterior wall, and one at the fundus of the uterus near cornual region. A false passage was seen in one patient. No uterine perforation occurred. There was no patient with failure of entry.

Table 3. The most common combination of movements found with respect to factors possibly affecting cervical canal direction.

\begin{tabular}{|c|c|c|c|c|c|}
\hline \multirow[t]{2}{*}{ Group } & & & \multicolumn{3}{|c|}{ Most common combination of movements preformed } \\
\hline & & & 1 & 2 & Others \\
\hline & & No (\%) & No (\%) & No (\%) & No $(\%)$ \\
\hline \multirow[t]{4}{*}{ Age } & $<25$ yrs & $40(16 \%)$ & DRUS $14(35 \%)$ & LRUS04 (10\%) & $22(55 \%)$ \\
\hline & $26-35$ yrs & $171(68.4 \%)$ & DRUS $110(64.3 \%)$ & DLUS17 (9.9\%) & $44(25.8 \%)$ \\
\hline & $>35 \mathrm{yrs}$ & $39(15.6 \%)$ & DRUS 31 (79.4\%) & DLUS06 (15.3\%) & $02(05 \%)$ \\
\hline & & \multicolumn{4}{|c|}{ chi-square $=26.2$ probability $=0.000$} \\
\hline \multirow[t]{4}{*}{ Parity } & 0 & $156(62.4 \%)$ & DRUS $94(60.2 \%)$ & DLUS14, $(8.9 \%)$ & $48(30.9 \%)$ \\
\hline & 1 & $46(18.3 \%)$ & DRUS $28(60.8 \%)$ & DLUS04 (8.6\%) & $14(30.6 \%)$ \\
\hline & $2+$ & $48(19.3 \%)$ & DRUS $33(68.7 \%)$ & DLUS06 (12.5\%) & $09(18.8 \%)$ \\
\hline & \multicolumn{5}{|c|}{ chi-square $=2.91$ probability $=0.573$} \\
\hline \multirow[t]{3}{*}{ Diagnosis $^{*}$} & No pathology & $36(14.4 \%)$ & DRUS $26(72.2 \%)$ & DLUS $02(5.5 \%)$ & $08(22.3 \%)$ \\
\hline & Pathology present & $214(85.6 \%)$ & DRUS $129(60.2 \%)$ & DLUS22 (10.2\%) & $63(29.44 \%)$ \\
\hline & \multicolumn{5}{|c|}{ chi-square $=1.99$ probability $=0.370$} \\
\hline \multirow[t]{3}{*}{ Previous * surgery on uterus } & YES & $76(30.8 \%)$ & DRUS $50(65.78 \%)$ & DLUS $10(13.15 \%)$ & $16(21.05 \%)$ \\
\hline & NO & $174(69.2 \%)$ & DRUS105 (60.30\%) & DLUS $(14,08.04 \%)$ & $55(31.66 \%)$ \\
\hline & \multicolumn{5}{|c|}{ chi-square $=3.77$ probability $=0.152$} \\
\hline \multirow[t]{3}{*}{$\begin{array}{l}\text { Force at Internal OS used or } \\
\text { scissors used before final entry }\end{array}$} & YES & $44(17.6 \%)$ & DRUS 27 (61.3\%) & DLUS 05 (11.3\%) & $12(27.27 \%)$ \\
\hline & NO & $206(82.4 \%)$ & DRUS $128(62.1 \%)$ & DLUS $19(9.2 \%)$ & $59(28.64 \%)$ \\
\hline & \multicolumn{5}{|c|}{ chi-square $=0.200$ probability $=0.905$} \\
\hline
\end{tabular}

DRUS-Down-Right-Up-Straight, LRUS-Left-Right-Up-Straight, DLUS-Down-Left-Up-Straight.

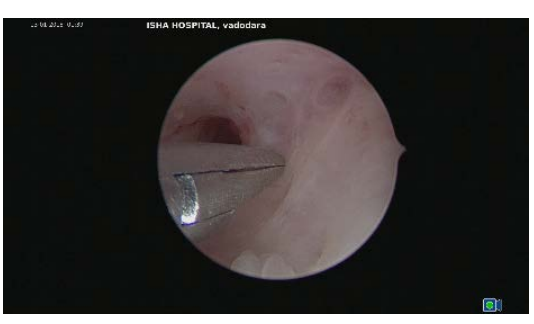

(a)

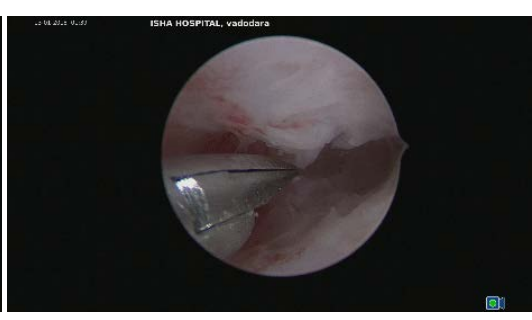

(b)

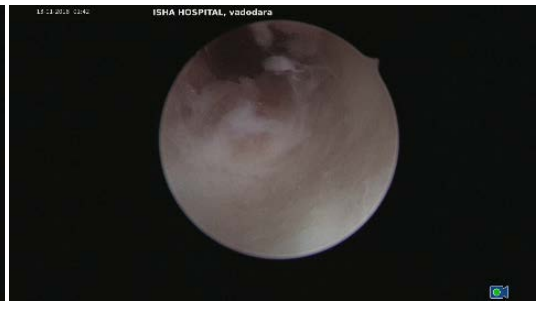

(c)

Figure 8. (a, b) Fibrosis at internal OS cut with scissors; (c) Fibrosis at internal OS opened. 


\section{Discussion}

In general, diagnostic and operative hysteroscopies have less complication rates, $<1 \%$. Uterine perforation is one of the most common complications of operative hysteroscopic surgeries, with an incidence of $0.12 \%-3 \%$ [3]. Majority of this published literature describes method using cervical dilation during the hysteroscopic procedure. Some single-center studies report higher complication rates than usual [4]. Many studies even mention use of slow cervical dilation to prevent cervical injury and uterine perforation. However, these slow dilation methods are also not safe in all [5] [6]. Using flexible or small diameter rigid dilators blindly through cervical canal for required the cervical dilation also can create more false passages or perforations [1]. In general publications appear to be describing the use of $4 \mathrm{~mm}$ telescope with diagnostic and resectoscope assembly to perform hysteroscopic surgeries. Ironically in some published studies, authors did not mention the size of hysteroscope used in their methods and they have discussed the use of methods to facilitate cervical dilation [7]. When the associated operative and resectoscope assembly are $>5 \mathrm{~mm}$ in diameter, cervical dilation is a must. Wide use of misoprostol to facilitate cervical dilation in the non-pregnant cervix may be associated with side effects and may not have optimum results [8] [9] [10] [11]. Use of smaller diameter telescopes $2.9 \mathrm{~mm}$ and less and associated operative and resectoscope assembly reduced the requirement of cervical dilation. Office hysteroscopy procedures use $1.9 \mathrm{~mm}$ diameter rigid scopes and related assembly [1].

The current study carefully tries to describe cervical canal directions found in hysteroscopic surgeries performed using 4-point directions in a patient placed in the standard lithotomy position. There is no information on the commonest directions of the cervical canal. S.H. Walker in 2018 had suggested a correct technique to position internal OS on a video screen while using 30-degree telescope. He advised to guide hysteroscope along the posterior wall of the cervix and to keep internal OS seen at 6 o'clock position on video in the anteverted uterus. This is to prevent perforation or false passage [12]. In the current study when the hysteroscopic sheath is placed at external OS in horizontal direction keeping the light cord at 12 o'clock, as per the method described the sheath has to advance downwards as first movement to see and then follow posterior wall of the cervix ( $2.9 \mathrm{~mm} \mathrm{12-degree} \mathrm{telescope).} \mathrm{On} \mathrm{further} \mathrm{advancing} \mathrm{the} \mathrm{sheath} \mathrm{following}$ the posterior wall of the cervical canal the internal OS position may be clearly visible now in the anterior part of the screen around 12'oclock position. Now the hysteroscopic sheath is further advanced and rotated to keep internal OS in anterior or central position. DRUS (downwards, right upwards, and finally straight) was the commonest direction observed in 155 (62.5\%) patients. This was followed by DLUS in 24 (9.6\%) patients.

As the number of patients is less in the age group $<25$ years it would not be possible to ascertain the commonest cervical canal direction. In this group of patients, the commonest cervical canal direction as DRUS was present only in 
about $1 / 3^{\text {rd }}$ of patients $14(35 \%)$. In age group $>26-35$ yrs. and $>35$ yrs the majority had DRUS as the commonest direction as was seen in $110(64.3 \%)$ and 31 (79.4\%) respectively.

The parity of the patient does not affect the commonest cervical direction reported as DRUS $(\mathrm{P}=0.573)$.

Similarly, the other variables as the presence of pelvic pathology, history of previous surgery on uterus performed or fibrosis observed at internal OS/resistance felt at internal OS did not seem to change the commonest observed cervical canal direction as DRUS. The observations found did not found any statistically significant difference with their association and common cervical canal direction observed. (Table 3) Having observed this it can be generally said that previous surgeries or associated pathologies would tend to change the uterine axis but the cervical canal direction is not affected.

In this study, we did not require ultrasound guidance in any case.

Cervical stenosis at internal OS because of fibrosis was overcome by using sharp scissors to cut the fibrosis, open the internal OS and now enter the uterine cavity. A similar approach is indicated in various studies [13] [14]. We do not recommend the use of electrosurgery to deal with such cervical stenosis and have found sharp tip scissors more effective. These methods were described when vaginoscopies and hysteroscopies became popular as office hysteroscopic procedures using smaller diameter hysteroscopes [2] [14].

Having known the most frequent direction of the cervical canal we have now found the blind insertion of foley's catheter and ET catheter, IUI cannula easier through the tortuous cervical canal in our clinical practice without holding cervix [15].

Limitation of the study: This was a single observer study. The cervical direction assessment was carried out by a definite technique keeping light cord at 12 o'clock position (2.9 mm 12-degree telescope) to begin with when hysteroscope was first positioned at external OS to define the cervical canal direction.

\section{Conclusion}

The study confirms the tortuous direction of the cervical canal. During the study period, having known the most common direction of cervical canal, blind insertion of cannula through cervical canal in the procedures as IUD insertion, IUI, embryo transfer, inserting foleys catheter for sonosalpingography etc. was easy. This was because now we knew the common cervical canal directions. The present study using the visual guided entry of hysteroscopic sheath through the cervical canal finds most frequent four point direction as DRUS, DURS and DLUS. Together these directions make the most frequent direction of cervical canal (found in $81.6 \%$ ). There was other interesting information, even in the presence of pelvic pathology and history of previous surgery these directions remain most frequent. These pathologies do not alter the cervical direction. In the current study group of hysteroscopic procedures, there are no case of failure of entry and no 
entry related complications. More publications are necessary concerning to cervical direction assessment by hysteroscopy or another method as MRI.

\section{Conflicts of Interest}

The authors declare no conflicts of interest regarding the publication of this paper.

\section{References}

[1] Sardo, A.D.S., Calagna, G. and Di Carlo, C. (2015) Tips and Tricks in Office Hysteroscopy. Gynecology and Minimally Invasive Therapy, 4, 3-7. https://doi.org/10.1016/j.gmit.2014.12.004

[2] Bettocchi, S., et al. (2016) Challenging the Cervix: Strategies to Overcome the Anatomic Impediments to Hysteroscopy: Analysis of 31,052 Office Hysteroscopies. Fertility and Sterility, 105, e16-e17. https://doi.org/10.1016/j.fertnstert.2016.01.030

[3] Jansen, F.W., Vredevoogd, C.B., Van Ulzen, K., Hermans, J., Trimbos, J.B. and Trimbos-Kemper, T.C.M. (2000) Complications of Hysteroscopy: A Prospective, Multicenter Study. Obstetrics \& Gynecology, 96, 266-270. https://doi.org/10.1097/00006250-200008000-00021

[4] Shveiky, D., Rojansky, N., Revel, A., Benshushan, A., Laufer, N. and Shushan, A. (2007) Complications of Hysteroscopic Surgery: "Beyond the Learning Curve". Journal of Minimally Invasive Gynecology, 14, 218-222. https://doi.org/10.1016/j.jmig.2006.07.019

[5] Wortman, M., et al. (2007) Complications of Hysteroscopic Surgery: "Beyond the Learning Curve”. Journal of Minimally Invasive Gynecology, 14, 530-531. https://doi.org/10.1016/j.jmig.2007.04.007

[6] Lichtenberg, E.S. (2004) Complications of Osmotic Dilators. Obstetrical and Gynecological Survey, 59, 528-536. https://doi.org/10.1097/00006254-200407000-00022

[7] Saha, M., Chakraborty, A., Chattopadhyay, S., Saha, S., Paul, J. and Das, A. (2015) Effect of Misoprostol for Cervical Priming before Gynecological Procedures on Nonpregnant Premenopausal Women. Journal of Natural Science, Biology and Medicine, 6, S123-S127. https://doi.org/10.4103/0976-9668.166116

[8] Shahraki, Z., Ganjali, Y. and Ghajarzadeh, M. (2019) Misoprostol and Isosorbide Mononitrate for Cervical Ripening before Hysteroscopy: A Randomized Clinical Trial. Maedica (Buchar), 14, 260-263

[9] Zhuo, Z., Yu, H., Gao, L. and Jiang, X. (2019) Effectiveness of Misoprostol Administration for Cervical Ripening in Women before Operative Hysteroscopy: A Randomized, Double-Blinded Controlled Trial. Minimally Invasive Therapy and Allied Technologies, 28, 344-350. https://doi.org/10.1080/13645706.2018.1559195

[10] Nakano, F.Y., et al. (2018) Efficacy of Misoprostol before Diagnostic Hysteroscopy in Postmenopausal Women: A Randomized Clinical Trial. Menopause, 25, 789-794. https://doi.org/10.1097/GME.0000000000001074

[11] Al-Fozan, H., Firwana, B., Al Kadri, H., Hassan, S. and Tulandi, T. (2015) Preoperative Ripening of the Cervix before Operative Hysteroscopy. Cochrane Database of Systematic Reviews, No. 4, CD005998. https://doi.org/10.1002/14651858.CD005998.pub2

[12] Walker, S.H. and Gokhale, L. (2018) Safety Aspects of Hysteroscopy, Specifically in Relation to Entry and Specimen Retrieval: A UK Survey of Practice. Gynecological 
Surgery, 15, 2. https://doi.org/10.1186/s10397-018-1036-6

[13] Wood, M.A., et al. (2018) Overcoming the Challenging Cervix: Identification and Techniques to Access the Uterine Cavity. Obstetrical and Gynecological Survey, 73, 641-649. https://doi.org/10.1097/OGX.0000000000000614

[14] Suen, M.W.H., Bougie, O. and Singh, S.S. (2017) Hysteroscopic Management of a Stenotic Cervix. Fertility and Sterility, 107, e19. https://doi.org/10.1016/j.fertnstert.2017.03.027

[15] Yanushpolsky, E.H., Ginsburg, E.S., Fox, J.H. and Stewart, E.A. (2000) Transcervical Placement of a Malecot Catheter after Hysteroscopic Evaluation Provides for Easier Entry into the Endometrial Cavity for Women with Histories of Difficult Intrauterine Inseminations and/or Embryo Transfers: A Prospective Case Series. Fertility and Sterility, 73, 402-405. https://doi.org/10.1016/S0015-0282(99)00492-6 